\title{
Glecaprevir/pibrentasvir for the treatment of chronic hepatitis C: design, development, and place in therapy
}

This article was published in the following Dove Press journal: Drug Design, Development and Therapy

\author{
Thomas G Cotter' \\ Donald $M$ Jensen ${ }^{2}$ \\ 'Center for Liver Diseases, The \\ University of Chicago Medicine, Chicago, \\ IL, USA; ${ }^{2}$ Section of Hepatology, RUSH \\ University Medical Center, Chicago, \\ IL, USA
}

\begin{abstract}
Direct-acting antiviral (DAA) therapy has changed the landscape of hepatitis C virus (HCV) management and has changed the focus to the possibility of $\mathrm{HCV}$ elimination in the near future. Glecaprevir, an NS3/4A protease inhibitor, and pibrentasvir, an HCV NS5A inhibitor, have addressed many of the existing shortcomings in the DAA therapy spectrum. This combination has proven to be a highly efficacious pan-genotypic DAA with a high barrier to resistance as a once-daily, all-oral medication. This review explores the design and development of glecaprevir and pibrentasvir, its place in current $\mathrm{HCV}$ management in the midst of a myriad of DAA therapy options, and also remaining challenges.
\end{abstract}

Keywords: glecaprevir, pibrentasvir, direct-acting antiviral therapy, hepatitis $\mathrm{C}$ virus

\section{Introduction}

It is estimated that over 70 million individuals have hepatitis $\mathrm{C}$ virus (HCV) viremia worldwide, with the incidence still increasing. ${ }^{1}$ If left untreated, chronic infection can lead to advanced liver disease with associated high morbidity and mortality rates from complications of decompensated disease, including hepatocellular carcinoma. ${ }^{2}$

Direct-acting antiviral (DAA) therapy has changed the landscape of HCV management and also of HCV-related liver transplantation (LT). Previously seen as a chronic, often incurable, infection, the highly effective DAA therapy has resulted in extremely high eradication rates and has changed the focus to the possibility of $\mathrm{HCV}$ elimination in the near future. ${ }^{3}$ In fact, the WHO has recently adopted the first "Global Health Sector Strategy on Viral Hepatitis, 2016-2021," which has a vision of eliminating viral hepatitis as a public health problem by globally reducing new viral hepatitis infections by $90 \%$ and reducing deaths due to viral hepatitis by $65 \%$ by $2030 .{ }^{4}$ In order to realize this goal, many challenges exist, not least the variability of many different genotypes (and subtypes) throughout the world. ${ }^{1}$

There are an estimated 3.5 million people in the United States with chronic HCV infection. ${ }^{5}$ Despite this, HCV is no longer the leading listing indication for LT in the United States, decreasing from 25.2\% to 17.6\% between 2006 and 2016, having been overtaken by non-alcoholic steatohepatitis and alcohol-related liver disease. ${ }^{6}$ Moreover, HCV-positive LT recipients in the DAA era have experienced improved 3-year graft survival, comparable to the outcomes of non-HCV-infected
Correspondence: Donald M Jensen Section of Hepatology, RUSH University Medical Center, 1725 West Harrison Street Suite 319, Chicago, IL 60612, USA Tel + I 3129428910

Email donald_m_jensen@rush.edu 
patients. ${ }^{7}$ In addition, the DAA era has afforded an opportunity to expand the use of HCV-positive liver donors, given the highly effective therapy now available, with increasing $\mathrm{HCV}$-positive liver grafts being transplanted into both $\mathrm{HCV}$-positive and $\mathrm{HCV}$-negative recipients. ${ }^{8}$ Similar trends have been noted in Europe with a notable rapid decline of LTs due to $\mathrm{HCV}$ infection and dramatically improved post-LT survival noted in the DAA era. ${ }^{9}$

Glecaprevir/pibrentasvir (GLE/PIB, AbbVie, North Chicago, IL, USA) is a fixed-dose combination of 2 DAA therapies and received Food and Drug Administration (FDA) approval on August 3, 2017, for the treatment of adult patients with chronic HCV genotype (GT) 1, 2, 3, 4, 5, or 6 infection without cirrhosis or with compensated cirrhosis. Additionally, it is indicated for adults with HCV GT 1 infection, who previously have been treated with an HCV
NS5A inhibitor or an NS3/4A protease inhibitor (PI). This review explores the design and development of GLE/PIB, examines its place in current $\mathrm{HCV}$ management in the midst of a myriad of DAA therapy options, and discusses the outstanding challenges in HCV treatment.

\section{Design and development Hepatitis C virology}

To understand the design and development of GLE/PIB, one must first examine the structure and lifecycle of the HCV. HCVis a positive-sense, single-stranded enveloped ribonucleic acid (RNA) virus. There are 7 major GTs (1-7), which vary by over $30 \%$ in nucleotide sequence from one another, and several subtypes (Figure 1). ${ }^{10}$ The natural course of infection can vary among these GTs, for example, GT 3 is

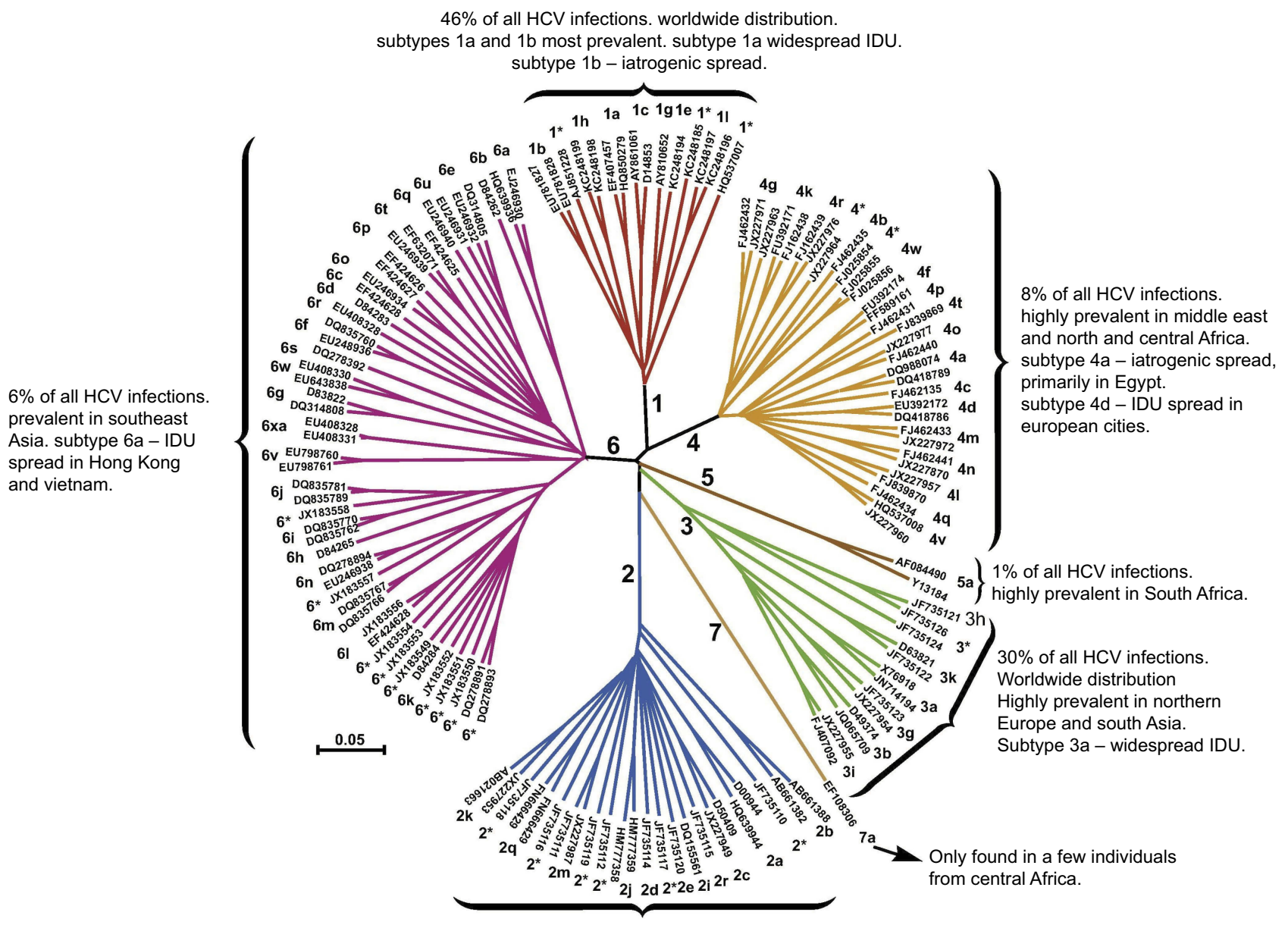

$9 \%$ of all HCV infections. worldwide distribution.

highly prevalent in western Africa. subtype $2 \mathrm{a}$ and $2 \mathrm{~b}$ most prevalent. subtypes $2 a$ and $2 b$ - iatrogenic spread.

Figure I Classification of hepatitis C virus into 7 major genotypes and subtypes. The tree is based on phylogenetic analysis of the open-reading frame (nucleotide) sequences. The overall prevalence and distribution are indicated for each major genotype.

Note: Reprinted from Journal of Hepatology, 65, Bukh J, The history of hepatitis C virus (HCV): basic research reveals unique features in phylogeny, evolution and the viral life cycle with new perspectives for epidemic control, S2-S2I, Copyright (2016), with permission from Elsevier."

Abbreviations: HCV, hepatitis C virus; IDU, intravenous drug use. 
associated with liver steatosis, increased fibrosis progression, and a higher risk of HCC, leading to difficulty in treating this subtype. HCV replicates primarily in hepatocytes of infected patients ${ }^{11}$ and has been found to be dependent on the liverspecific microRNA-122. ${ }^{12}$ Extrahepatic replication of HCV has been recognized, and there had been a concern in the preDAA era that persistence of HCV replication at these sites could potentially play a role in late recurrence after treatment. In the pre-DAA era, it had been shown that in most patients with sustained virologic response (SVR), low-level HCV RNA can be detected in lymphocytes and monocytes/macrophages, and occasionally in liver and serum for up to 9 years after the end of therapy. ${ }^{13}$

Genotypes 1 and 3 are the most prevalent worldwide, accounting for $46 \%$ and $30 \%$ of all infections, respectively, while GTs $2,4,5$, and 6 account for $9 \%, 8 \%, 1 \%$, and $6 \%$, respectively (Figure 1). ${ }^{14}$ Some GTs show a high degree of endemicity, notably GT 3 in Pakistan and India and GT 4 in the Middle East and Egypt (Figure 1). ${ }^{15,16}$ In addition, there has been changing geographical distribution of GTs and subtypes over time due to eradication of transfusion-associated transmission, increasing intravenous drug abuse, and population migration patterns. For example, subtypes $1 \mathrm{~b}, 2 \mathrm{a}$, and $2 \mathrm{~b}$ are typically associated with elderly populations thought to be related to transfusion exposures, while other subtypes, such as $1 \mathrm{a}, 3 \mathrm{a}, 4 \mathrm{~d}$, and $6 \mathrm{a}$, are linked with intravenous drug abuse. ${ }^{17}$

The HCV genome has one continuous open-reading frame flanked by nontranslated regions (NTRs) at $5^{\prime}$ and $3^{\prime}$ ends. The $5^{\prime}$ NTR also contains the internal ribosome entry site that initiates the cap-independent translation of $\mathrm{HCV}$ genome into a single 3000 amino acid polyprotein, ${ }^{18}$ which is cleaved by a combination of viral and host proteases into 10 mature viral proteins - divided into structural and nonstructural (NS) proteins (Figure 2). ${ }^{11}$ The structural proteins consist of core and envelope. $\mathrm{HCV}$ core is the viral nucleocapsid protein with several functions including RNA binding, immune modulation, cell signaling, autophagy, and assisting with $\mathrm{HCV}$ assembly. ${ }^{11}$ HCV E1/E2 are glycosylated envelope glycoproteins that surround the viral particles, protecting it from immunological responses and contributing to $\mathrm{HCV}$ persistence. $^{19}$

The identification of the NS (p7, NS2, NS3, NS4A, NS4B, NS5A, and NS5B) proteins and their recognition as therapeutic targets were important therapeutic breakthroughs in HCV management. The ion channel protein p7 is downstream of the envelope region and contributes to viral assembly and release. NS2 is a viral autoprotease that mediates the cleavage between NS2 and NS3, thus having a vital role in viral assembly. The NS3 and NS4A viral proteins act synergistically to perform a serine-type protease function, resulting in the cleavage of the $\mathrm{HCV}$ viral polyprotein at four sites. ${ }^{20}$ The role of NS4B is not well understood, although it is known to induce the membranous web formation. The NS5A is a dimeric zincbinding metalloprotein which binds the viral RNA and various host factors in close proximity to $\mathrm{HCV}$ core and lipid droplets and is essential for RNA replication and the assembly of infectious virions, although the precise mechanism is unknown. ${ }^{21}$ Last, NS5B is an RNA-dependent RNA polymerase. Altogether, these proteins contribute to various aspects of $\mathrm{HCV}$ life cycle, including viral attachment, entry and fusion, HCV RNA translation,

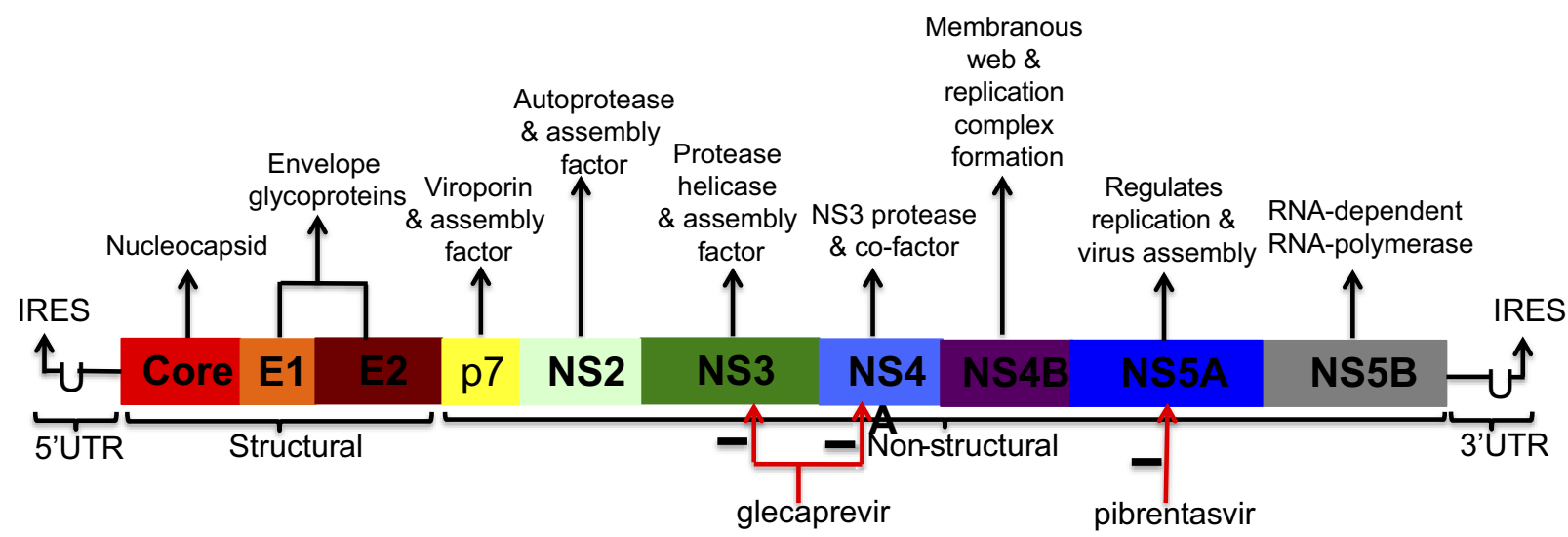

Figure 2 The structure and replication cycle of the hepatitis $C$ virus. The identification of the nonstructural proteins was an important therapeutic breakthrough. The sites of action of glecaprevir and pibrentasvir are shown.

Abbreviations: IRES, internal ribosome entry site; E, envelope; NS, nonstructural. 
posttranslational processing, $\mathrm{HCV}$ replication, virus assembly, and release. Of note, due to the highly errorprone RNA polymerase, $\mathrm{HCV}$ also displays remarkable genetic diversity and propensity for selection of immune evasion or drug resistance mutations.

\section{DAA therapy and the rationale for glecaprevir/pibrentasvir}

In the DAA era, there are three broad pharmacological groups which act in combination targeting multiple components of the $\mathrm{HCV}$ viral replication process: NS3/4A protease inhibitors (PIs), NS5A replication complex inhibitors, and NS5B polymerase inhibitors (Table 1).

The first DAAs were approved for combination use with peginterferon alfa and ribavirin in May 2011 by the US FDA. These NS3/4A protease inhibitors, boceprevir and telaprevir, achieved an SVR of up to $68 \%$ and $75 \%$, respectively, in untreated chronic HCV GT 1 infection. $^{22,23}$ However, high rates of adverse events, drug-drug interactions, and an inherent low barrier to viral resistance limited their overall impact. ${ }^{24}$ In November 2013, another NS3/4A PI, simeprevir, was FDA approved for HCV GT 1 treatment in combination with peginterferon alfa and ribavirin and achieved an 80\% 12-week SVR (SVR12). ${ }^{25}$ Sofosbuvir, an NS5B polymerase inhibitor, was then approved by the FDA in December 2013, after demonstrating an SVR12 of 90\% in GTs 1 and 4 infection in combination with peginterferon alfa and ribavirin. ${ }^{26}$

Subsequently, the combination use of DAAs has resulted in highly effective interferon-free regimens. As an example, combined simeprevir and sofosbuvir treatment has resulted in SVR12s of 73-97\%, with higher cure rates obtained in those without cirrhosis and those treated for 12 weeks as opposed to 8 weeks. ${ }^{27-29}$ Combined ledipasvir and sofosbuvir has achieved 94-100\% SVR12 rates for treatment-naïve (TN) patients and $87-100 \%$ SVR12 rates for treatment-experienced (TE) patients. ${ }^{30,31}$

Despite these rapidly evolving and highly effective treatments, a number of challenges remained. Virologic failures of DAA regimens were increasing and thought to be related to baseline resistance-associated polymorphisms or resistanceassociated substitutions (RASs) that emerged during therapy. ${ }^{32}$ As our understanding increased, it was observed that variants within the NS5A region markedly increased the risk of treatment failure. ${ }^{33}$ Retreatment strategies were also inadequate, with no treatments specifically indicated for either NS5A or NS5B inhibitor-experienced patients. As an example, retreatment with ledipasvir/sofosbuvir demonstrated only an $18 \%$ SVR12 in patients with prior nonresponse to NS5A inhibitors regimens. ${ }^{34}$ Although much improved SVR12 was noted with the addition of ribavirin to ledipasvir/sofosbuvir. ${ }^{35}$ Moreover, none of the existing DAA options was approved for patients with advanced chronic kidney disease (CKD), a major shortcoming considering the high prevalence of $\mathrm{HCV}$ in this patient population. Last, most of the approved regimens recommended 12 weeks of treatment; however, there was concern of declining patient adherence during the last 4 weeks of treatment, suggesting that shorter treatment durations may improve adherence particularly in difficult-to-treat population such as psychiatric patients and the prison population. ${ }^{36}$

Therefore, a highly effective pan-genotypic DAA for patients with $\mathrm{HCV}$ without the requirement of baseline genotyping, viral load, or resistance testing and with shorter treatment duration and safe in advanced renal disease was highly sought after, with the ideal of simplifying future treatment algorithms.

\section{Pharmacology of glecaprevir and pibrentasvir}

In order to attempt to address the shortcomings in the existing HCV-treatment market, GLE/PIB was developed. Glecaprevir's (identified by AbbVie and Enanta) molecular formula is $\mathrm{C}_{38} \mathrm{H}_{46} \mathrm{~F}_{4} \mathrm{~N}_{6} \mathrm{O}_{9} \mathrm{~S}$, and its chemical structure is shown in Figure $3 .{ }^{37}$ It is an HCV NS3/4A PI, preventing the proteolytic cleavage of the $\mathrm{HCV}$-encoded polyprotein into mature forms of the NS3, NS4A, NS4B, NS5A, and NS5B proteins, and thus preventing viral replication. In a preapproval biochemical assay, glecaprevir inhibited the proteolytic activity of recombinant NS3/4A enzymes from clinical isolates of HCV GTs 1a, 1b, 2a, 2b, 3a, 4a, 5a, and $6 a^{37}$ Moreover, it demonstrated $<5$-fold loss of activity against common GT 1 variants at key resistance-associated positions of R155 and D168 to existing NS3/4A PIs. ${ }^{37}$ It is readily oral bioavailable with time for peak plasma concentration (Tmax) at $5 \mathrm{hrs}$. It is predominantly protein bound, undergoes secondary metabolism via cytochrome P450 (CYP) 3A, and has an elimination half-life $\left(t_{1 / 2}\right)$ of $6 \mathrm{hrs}$, with the biliary-fecal route being the major route of excretion. ${ }^{37}$

Pibrentasvir's molecular formula is $\mathrm{C}_{57} \mathrm{H}_{65} \mathrm{~F}_{5} \mathrm{~N}_{10} \mathrm{O}_{8}$, and its chemical structure is shown in Figure 4. It is an HCV NS5A inhibitor, preventing viral RNA replication and virion assembly. Half-maximal effective concentration (EC50) values are $\leq 5$ picomolar across all major HCV GTs and maintain high potency against common NS5A 


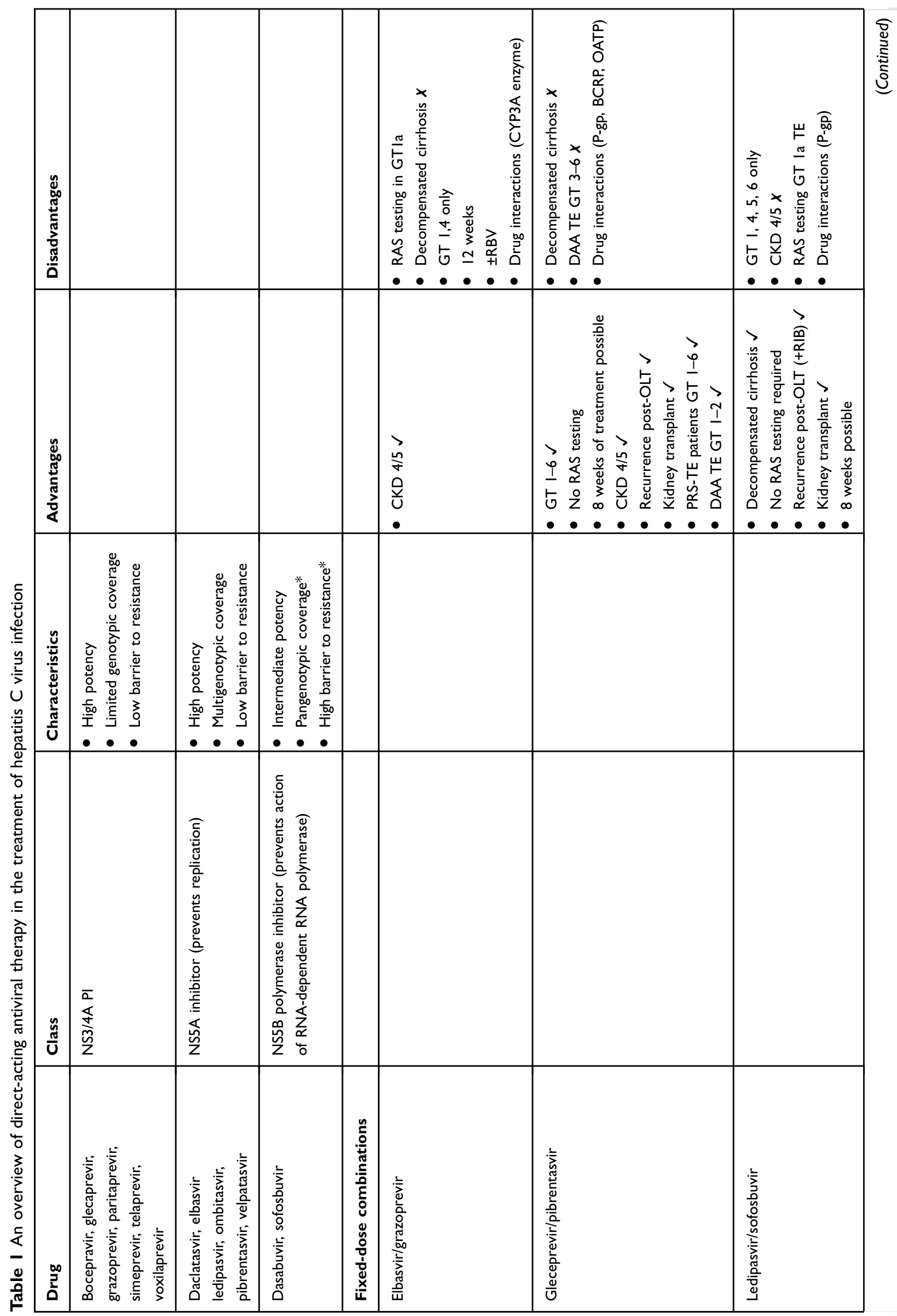




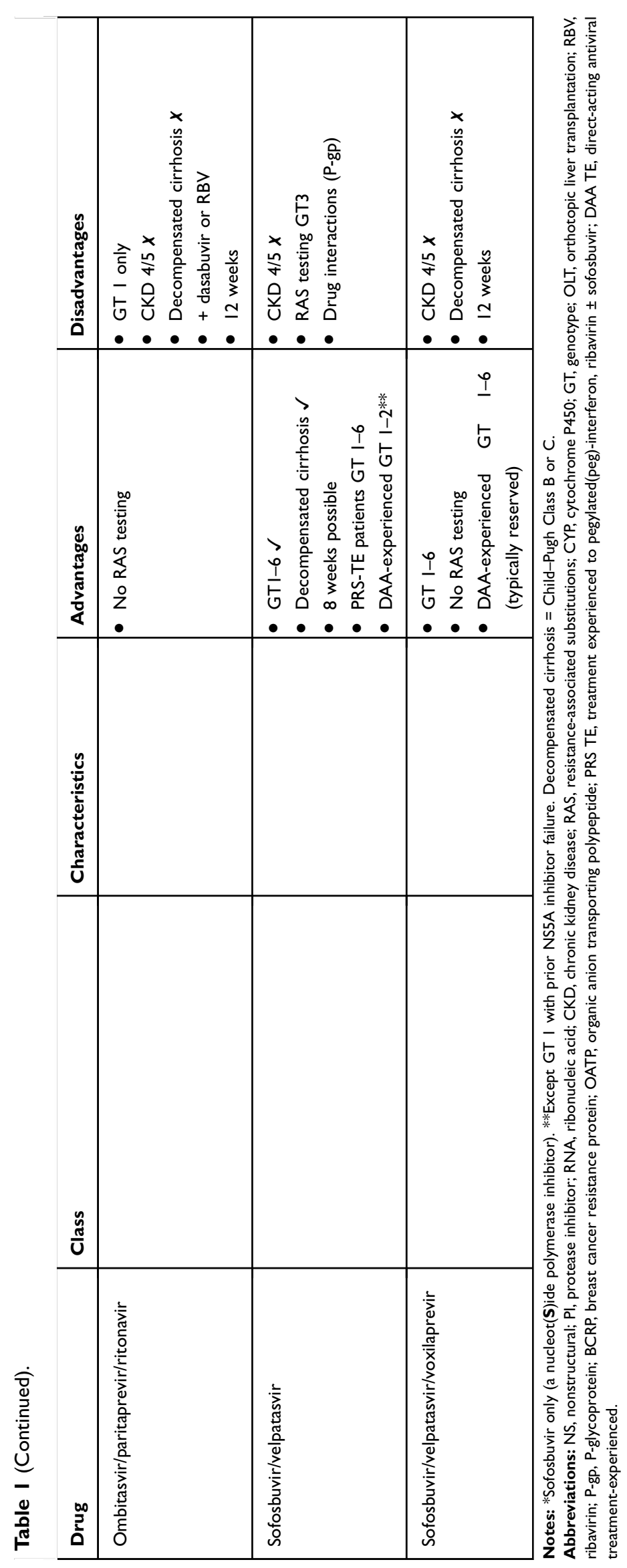




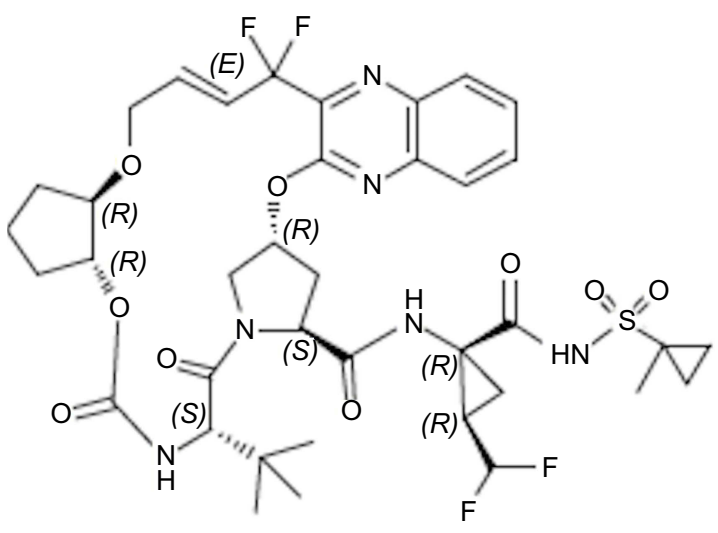

Figure 3 The chemical structure of glecaprevir (C38H46F4N6O9S).

resistance-associated variants, including GT1a Y93H (6.7fold increase in EC50), ${ }^{38}$ which has been associated with reduced susceptibility to other NS5A inhibitors. ${ }^{39}$ Pibrentasvir has a Tmax of $5 \mathrm{hrs}$ when taken orally. It is predominantly protein bound, does not undergo metabolism, and has an elimination $t_{1 / 2}$ of $13 \mathrm{hrs}$, with the biliaryfecal route again being the major route of excretion. ${ }^{37}$

Both glecaprevir and pibrentasvir are inhibitors of P-glycoprotein and organic anion transporting polypeptide 1B1/3, as well as being weak inhibitors of CYP3A, 1A2, and uridine glucuronosyltransferase $1 \mathrm{~A} 1$, which leads to a number of important potential drug interactions. Co-administration of carbamazepine, phenytoin, rifampin, efavirenz, and St. John's wort may significantly decrease the plasma concentrations of GLE/PIB, and the use of these medications with GLE/ $\mathrm{PIB}$ is not recommended. Other potentially significant drug interactions include GLE/PIB increasing the levels of digoxin, statins, and dabigatran, while conversely, cyclosporine increases GLE/PIB levels. $^{37}$

There is not enough safety data to support GLE/PIB use in pregnancy. Its safety in children $<18$ years of age has not been established. No dosage adjustment is required in $\mathrm{CKD}$, including patients on dialysis, while GLE/PIB is contraindicated in severe hepatic impairment (Child-Pugh C). ${ }^{37}$

In summary, when used in combination, GLE/PIB has pan-genotypic anti-HCV activity including against resistant strains, minimal metabolism, primarily biliary excretion, and negligible renal excretion. The next step was to assess its performance in human clinical trials.

\section{Registrational studies of glecaprevirl pibrentasvir in HCV treatment}

The registrational clinical trials of glecaprevir/pibrentasvir are summarized in Table 2.

\section{HCV GT I, 2, 4, 5, or 6 infection without cirrhosis}

The efficacy of GLE/PIB was assessed in TN or (peg)interferon, ribavirin, and/or sofosbuvir (PRS)-TE adult patients with HCV GT 1, 2, 4, 5, or 6 infection without cirrhosis in 4 trials using 8- or 12-week durations: ENDURANCE-1, ENDURANCE-4, SURVEYOR-1 (Part 2), and SURVEYOR-2 (Part 2 and Part 4). ENDURANCE-1 was a randomized, open-label trial comparing 8 and 12 weeks of treatment with GLE/PIB in patients with GT 1 infection. ${ }^{40}$ There was a 99\% (348/351) SVR12 in the 8-week treatment group, which was numerically similar to the 12 -week treatment group outcome. Pooled analysis from the other 3

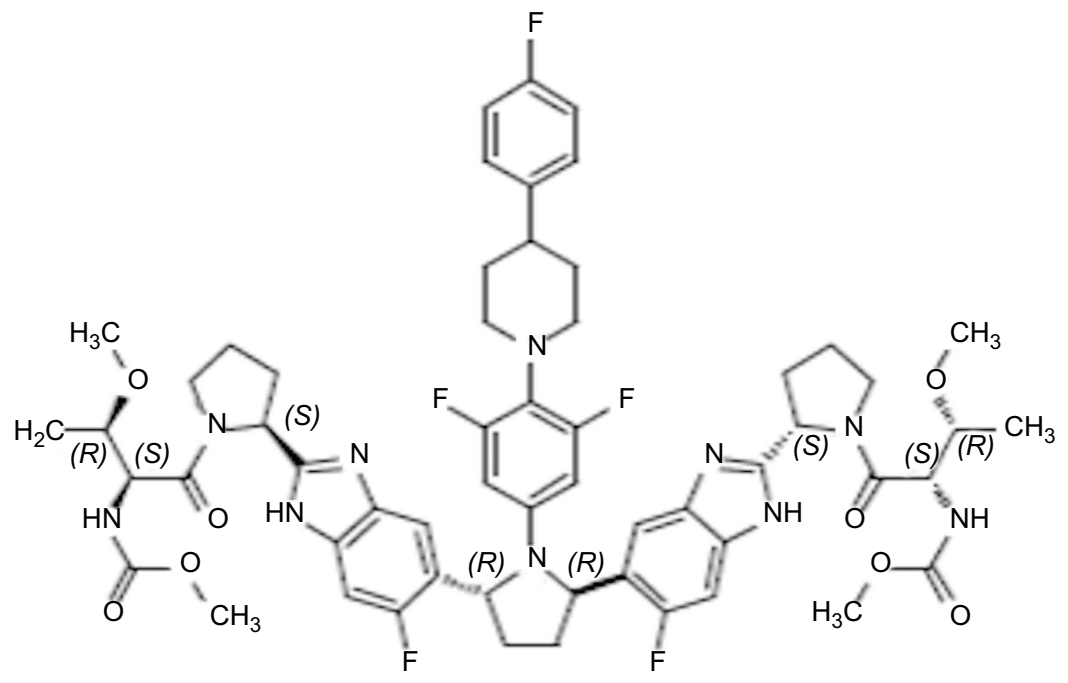

Figure 4 The chemical structure of pibrentasvir (C57H65F5NI0). 
Table 2 Overview of the clinical trials evaluating the efficacy of glecaprevir/pibrentasvir in patients with HCV genotype I, 2, 3, 4, 5, or 6 infection, according to treatment history and cirrhosis status

\begin{tabular}{|c|c|c|c|}
\hline TRIAL & Genotype & Treatment duration & I 2-SVR rate (\%) \\
\hline \multicolumn{4}{|l|}{ TN and PRS-TE patients without cirrhosis } \\
\hline ENDURANCE-I & I & $8(n=351)$ or 12 weeks $(n=352)$ & 99 \\
\hline SURVEYOR-2 & 2 & 8 weeks $(n=197)$ & 98 \\
\hline ENDURANCE-3 & 3 & $8(n=157)$ or 12 weeks $(n=233)$ & $94.9,95.3$ \\
\hline SURVEYOR-2 & 3 & I6 (PRS-TE only) weeks ( $n=22$ ) & $96-98$ \\
\hline ENDURANCE-4/SURVEYOR-I/SURVEYOR-2 & $4,5,6$ & 12 weeks $(n=245)$ & $93-100$ \\
\hline ENDURANCE-4/SURVEYOR-I/SURVEYOR-2 & $4,5,6$ & 8 weeks $(n=10)$ & 100 \\
\hline \multicolumn{4}{|c|}{ TN and PRS-TE patients with compensated cirrhosis } \\
\hline EXPEDITION-I & I, $2,4,5,6$ & 12 weeks $(n=146)$ & 99 \\
\hline SURVEYOR-2 & 3 & 12 weeks (TN) or 16 weeks (PRS-TE) & $96-98$ \\
\hline \multicolumn{4}{|c|}{ Patients with CKD stage 4 and $5 \pm$ compensated cirrhosis } \\
\hline EXPEDITION-4 & $1-6$ & 12 weeks $(n=104)$ & 98 \\
\hline \multicolumn{4}{|c|}{ NS5A inhibitor or PI-experienced patients \pm compensated cirrhosis } \\
\hline MAGELLAN-I & I & 12 or 16 weeks & $86-100$ \\
\hline \multicolumn{4}{|c|}{ HCV-HIV-I co-infected patients \pm compensated cirrhosis } \\
\hline EXPEDITION-2 & $\mathrm{I}, 2,3,4,6$ & 8 or 12 weeks $(n=153)$ & 98 \\
\hline \multicolumn{4}{|c|}{ Liver or kidney transplant recipients without cirrhosis } \\
\hline MAGELLAN-2 & I, $2,3,4,6$ & 12 weeks $(n=100)$ & 98 \\
\hline
\end{tabular}

Abbreviations: $n$, sample size; TN, treatment naïv; PRS-TE (peg)interferon, ribavirin \pm sofosbuvir-treatment experienced; SVR, sustained virologic response; CKD, chronic kidney disease; $\mathrm{HCV}$, hepatitis $\mathrm{C}$ virus; NS5A, nonstructural protein $5 \mathrm{~S}$.

clinical trials (ENDURANCE-4, SURVEYOR-1, and SURVEYOR-2) $)^{41,42}$ showed SVR12 rates of $98 \%$ $(193 / 197), 93 \%(43 / 46), 100 \%(2 / 2)$, and 100\% (10/10) in GT 2, 4, 5, and 6 patients treated for 8 weeks, respectively. While SVR12 rates were 100\% (27/27) and 100\% (30/30) in GT 5 and 6 patients treated for 12 weeks, respectively.

\section{HCV GT I, 2, 4, 5, or 6 infection with compensated cirrhosis}

The effectiveness of GLE/PIB in TN or PRS-TE patients with chronic HCV GT 1, 2, 4, 5, or 6 infection with compensated cirrhosis (Child-Pugh A) was studied in the single-arm, open-label EXPEDITION-1 trial. ${ }^{43}$ Of the 146 patients treated, 145 (99\%) achieved SVR12 after 12 weeks of treatment. The 1 patient who did not respond was GT 1.

\section{HCV GT 3 infection without and with compensated cirrhosis}

The effectiveness of GLE/PIB in TN or PRS-TE patients with chronic HCV GT 3 infection without cirrhosis or with compensated cirrhosis was explored in ENDURANCE-3 and in SURVEYOR-2 (Part 3). ENDURANCE-3 was a partially randomized, openlabel, active-controlled trial in TN subjects. ${ }^{40}$ Patients were randomized to either 12 weeks of GLE/PIB or sofosbuvir/daclatasvir. The trial included a third nonrandomized arm with GLE/PIB for 8 weeks. The SVR12 was 94.9\% (149/157) and 95.3\% (222/233) in the 8- and 12-week glecaprevir/pibrentasvir treatment groups, respectively. Notably, among those treated for 8 weeks, only $75 \%(12 / 16)$ with the A30K RAS at baseline achieved SVR, although such patients represented a minority $(<7 \%)$ of the study population. SURVEYOR-2 was an open-label trial randomizing PRS-TE patients with GT 3 infection without cirrhosis and with compensated cirrhosis to 12 or 16 weeks of treatment. $^{42}$ TN patients with cirrhosis achieved a $98 \%$ (39/40) SVR12 after 12 weeks of treatment, while PRS-TE patients \pm cirrhosis achieved a 96\% (66/99) SVR12 after 16 weeks of treatment. 


\section{NS5A inhibitor and/or NS3/4A PI- experienced patients}

In MAGELLAN-1, the efficacy of 12 weeks of GLE/PIB in patients with prior treatment failure of regimens containing an NS5A inhibitor and/or NS3/4A PI \pm NS5B inhibitors was evaluated. The trial was a randomized, openlabel trial in 141 GT 1- or 4-infected patients. Part 1 $(n=50)$ was a randomized trial exploring 12 weeks of glecaprevir $200 \mathrm{mg}$ and pibrentasvir $80 \mathrm{mg}$ (arm A), glecaprevir $300 \mathrm{mg}$ and pibrentasvir $120 \mathrm{mg}, \pm$ ribavirin (arms B \& C). ${ }^{44}$ The results showed SVR12 was achieved in $100 \%(6 / 6), 95 \%(21 / 22)$, and $86 \%(19 / 22)$ of patients in $\operatorname{arms} \mathrm{A}, \mathrm{B}$, and $\mathrm{C}$, respectively. Part $2(\mathrm{n}=91)$ randomized GT 1- or 4-infected patients without cirrhosis or with compensated cirrhosis to 12 or 16 weeks of treatment with GLE/PIB. ${ }^{45}$ SVR 12 was achieved by $89 \%$ (39 of 44 ) and $91 \%$ (43 of 47) of patients who received 12 and 16 weeks of GLE/PIB, respectively. Virological relapse occurred in $9 \%$ (4 of 44) of the patients treated with 12 weeks of GLE/PIB, with no relapses in the 16 weeks of the treatment group. Past treatment history with one class of inhibitor (protease or NS5A) had no impact on SVR12, whereas past treatment with both classes of inhibitors was associated with lower SVR12 rates.

\section{Special groups}

EXPEDITION-4 was an open-label, single-arm trial assessing the safety and efficacy of GLE/PIB in patients with CKD Stages 4 and 5 without cirrhosis and with compensated cirrhosis. ${ }^{46}$ In this cohort, there were 104 patients $(81 \%$ without cirrhosis and $42 \%$ PRS-TE), of whom $82 \%$ were on hemodialysis, and $53 \%, 15 \%, 11 \%, 19 \%, 1 \%$, and $1 \%$ were infected with HCV GTs 1, 2, 3, 4, 5, and 6, respectively. The overall SVR12 rate was $98 \%$ and there were no virologic failures. Importantly, renal impairment did not affect the efficacy and no dose adjustments were required.

EXPEDITION-2 was an open-label study in $153 \mathrm{HCV} /$ HIV-1-coinfected TN or PRS-TE patients without (8 weeks of treatment) or with compensated cirrhosis (12 weeks of treatment). ${ }^{47}$ The SVR12 rate was $98 \%$ (150/ 153). One subject experienced on-treatment virologic failure and no subjects relapsed.

MAGELLAN-2 was a single-arm, open-label study in 100 liver or kidney transplant recipients without cirrhosis who were infected with HCV GTs $1,2,3$, 4, or $6 .{ }^{48}$ The study included HCV TN or PRS-TE patients and patients were treated for 12 weeks. Immunosuppressants allowed for co-administration were cyclosporine $\leq 100 \mathrm{mg}$, tacrolimus, sirolimus, everolimus, azathioprine, mycophenolate, prednisone, and prednisolone. The overall SVR12 rate was $98 \%(98 / 100)$. There was one relapse and no on-treatment virologic failures.

GLE/PIB was well tolerated in all these registrational studies with a low rate of serious adverse events and withdrawal due to adverse events (Table 3).

\section{Discussion}

As a result of the aforementioned registrational clinic studies, GLE/PIB became the first pan-genotypic regimen approved for 8 weeks in TN, noncirrhotic patients and the only 8 -week regimen for patients co-infected with HIV. It also addressed the void of pan-genotypic options for patients with chronic $\mathrm{HCV}$ and advanced $\mathrm{CKD}$, including

Table 3 Summary of the adverse events of glecaprevir and pibrentasvir from Registrational Clinical Trials ${ }^{\mathrm{a}}$

\begin{tabular}{|c|c|}
\hline Most common AEs & Rate (\%) \\
\hline Headache & $6.7-25.8$ \\
\hline Fatigue & $7.0-24.0$ \\
\hline Nausea/vomiting & $3.3-13.7$ \\
\hline Asthesia & $0.0-10.0$ \\
\hline Pruritis & $0.0-20.0$ \\
\hline Diarrhea & $0.0-11.5$ \\
\hline Abdominal pain & $0.0-7.0$ \\
\hline Dizziness & $0.0-6.0$ \\
\hline URTI & $0.0-15.2$ \\
\hline Back pain & $0.0-9.0$ \\
\hline Constipation & $0.0-9.0$ \\
\hline Lethargy & $0.0-7.0$ \\
\hline Stomatitis & $0.0-1.1$ \\
\hline Malaise & $0.0-5.6$ \\
\hline Bilirubin increase & $0.0-1.1$ \\
\hline Insomnia & $0.0-6.0$ \\
\hline SAEs & $0.8-7.5$ \\
\hline WDAEs & $0.3-3.8$ \\
\hline
\end{tabular}

Notes: ${ }^{\mathrm{a} C}$ anadian Agency for Drugs and Technologies in Health. Clinical Review Report: Glecaprevir/Pibrentasvir (Maviret): (AbbVie Corporation; North Chicago, IL, USA): Indication: Hepatitis C genotype I to 6. Ottawa (ON), Feb 2018. Abbreviations: AEs, adverse events; SAEs, serious adverse events; WDAEs, withdrawals due to adverse events. 
dialysis patients. Following on from the index clinical trials, there have been a number of subsequent studies, including real-world data, which has helped to consolidate the place of GLE/PIB in HCV treatment and understand its role in certain clinical situations, such as previous treatment failures, elderly patients, and HCV GT 3 infection.

The efficacy and safety of GLE/PIB in patients with chronic HCV GT 5 or 6 infection was confirmed in the ENDURANCE-5, 6 trials. $^{49}$ In this study, 22/23 and 60/61 patients achieved SVR12 in GT5 and GT6 infections, respectively. The safety and efficacy of GLE/PIB have also been shown in patients aged 65 years or older with chronic $\mathrm{HCV}$ infection. In a study on 328 patients aged $\geq 65$ years, the SVR12 rate of $97.9 \%$ was not significantly different from the $97.3 \%$ SVR12 rate in patients $<65$ years $(p=0.555) .{ }^{50}$ In addition, the rate of AEs was similarly rare between the groups $(<0.5 \%)$.

The treatment of HCV GT 3 infection in patients with cirrhosis and/or prior treatment failure has remained challenging. Subsequent to the ENDURANCE-3 and SURVEYOR-2 (Part 3) trials, a real-world study from Italy on GLE/PIB demonstrated an SVR12 rate of $99.3 \%$ and excellent safety profiles in 723 patients with chronic $\mathrm{HCV}$, although there were notably lower SVR rates in HCV GT3 patients treated for 8 weeks. ${ }^{51}$ Pooled data from five Phase 2 or 3 trials that evaluated 8-, 12-, and 16-week GLE/PIB in 693 patients with chronic HCV GT3 infection demonstrated SVR12 in 95\% of the TN patients without cirrhosis receiving 8-week and 12week GLE/PIB. ${ }^{52}$ TN patients with cirrhosis had a 97\% SVR12 rate with 12-week GLE/PIB. Treatment-experienced (PRS), noncirrhotic patients had SVR rates of 90\% and $95 \%$ with 12- and 16-week GLE/PIB, respectively, while 94\% of the TE patients with cirrhosis treated for 16 weeks achieved SVR12. With this in mind, a 16-week course of GLE/PIB should be considered for TE patients with HCV GT 3 infection irrespective of the presence of cirrhosis. Furthermore, 12 weeks may be more prudent than 8 weeks for TN patients with HCV GT3 infection and difficult-to-treat characteristics, such as progressive fibrosis or preexisting A30K mutations.

There has been ongoing research aimed at ascertaining the appropriate management of TE patients with $\mathrm{HCV}$ infection, with duration ranging from 8 to 16 weeks depending on HCV GT and specific agents previously received. As noted earlier in the registrational clinical trials, PRS-TE patients can be effectively treated with GLE/PIB. However, in patients previously treated with a class of medication other than PRS, the indications for GLE/PIB are somewhat restricted. These indications do not include
NS5A or NS3/4A PI-experienced patients with GT 2-6 or HCV GT 1 who have received both NS5A and NS3/4A inhibitors. Approval has been restricted to patients infected with GT1, given the few patients with GT4 enrolled in MAGELLAN-1 trial. Salvage therapy with GLE/PIB was not recommended in patients with prior failure of both NS3/ 4A and NS5A inhibitors, after past treatment with both classes of inhibitors was associated with a significant lower SVR12 rate in MAGELLAN-1. These are notable limitations of GLE/PIB therapy. At this time, sofosbuvir, velpatasvir, and voxilaprevir (SOF/VEL/VOX) appear to hold a unique position in the treatment of patients with prior DAA failures on the basis of data from the POLARIS-1 and POLARIS-4 trials which showed no association between baseline RASs and treatment outcomes. ${ }^{53}$

In a real-world study in 30 patients with chronic $\mathrm{HCV}$ infection with prior DAA therapy failures, 28/30 (93.3\%) achieved SVR12 with GLE/PIB. ${ }^{54}$ Liver fibrosis stage did not affect efficacy; however, the results of this study suggested that some GT 1b HCV-infected patients, particularly those with NS5A-P32 deletion, may have low susceptibility to GLE/PIB. Additionally, another study found NS5A-P32 deletion to be a factor involved in virologic failure in patients receiving GLE/PIB. ${ }^{55}$ More extensive analysis has occurred assessing the affect on baseline polymorphisms (BPs) on GLE/PIB efficacy in order to assess if these need to be assessed for in everyday clinical practice. The resistance analysis of 2200 patients with HCV GT 1-6 infection from Phase 2 and 3 clinical trials showed that BPs in NS3 and/or NS5A did not have an impact on efficacy for patients with GT1-GT6, with the exception of TE GT3-infected patients treated for 12 weeks (as noted earlier). ${ }^{56}$ This led the authors to conclude that the GLE/PIB regimen, when the recommended durations are used, allows for a pan-genotypic treatment option without the need for baseline resistance testing. This represents a major advantage over the previous practice of resistance testing prior to grazoprevir/elbasvir and extensive use of ribavirin with either paritaprevir/ombitasvir/dasabuvir or LDV/SOF in TE patients with and without cirrhosis.

There are a number of remaining challenges and limitations of GLE/PIB at the present time. As with all other regimens containing $\mathrm{HCV} \mathrm{NS} 3 / 4 \mathrm{~A}$ PIs, GLE/PIB is not recommended in patients with decompensated cirrhosis (Child-Turcotte-Pugh stage B or C). This means that patients with decompensated cirrhosis and prior DAA failure have no explicit treatment options at this time. For these patients, alternative treatment options, including off-label DAA combinations without an NS3/4A inhibitor such as SOF/VEL 
\pm ribavirin, can be considered. Alternatively, treatment can be completed post-LT if the patient is a transplant candidate. There is uncertainty about the approach to patients with prior GLE/PIB failure, with one approach of 16 weeks of GLE/PIB plus SOF and ribavirin shown to be effective, ${ }^{57}$ alternatively treatment with SOF/VEL/VOX has also been suggested. ${ }^{53}$ One of the remaining challenges is the treatment of $\mathrm{HCV}$ in children, given the lack of availability of most DAAs for this age group. Sofosbuvir/ledipasvir and sofosbuvir/daclatasvir have been shown to be safe and effective in children. ${ }^{58}$ There is currently no data available on GLE/PIB on children with HCV. Only SOF/LDV and SOF plus ribavirin are approved for children aged 12 and over, whereas treatment in younger patients is recommended to be deferred until interferon-free regimens are approved. ${ }^{59}$ Finally, the lack of African American (4-5\%) and Hispanic (negligible) participants among TN patients limited the generalizability of GLE/ PIB from the registrational clinical trials, a deficit which has not been fully addressed by subsequent real-world data. Therefore, the majority of the existing data are more supportive of treating a European rather than a US population.

The existing body of evidence has prompted the FDA to approve GLE/PIB for the treatment of adult patients with chronic HCV GT 1-6 infection without cirrhosis (8 weeks of treatment) or with compensated cirrhosis (12 weeks of treatment), and for patients with HCV GT 1 infection, who previously have been treated with an NS5A inhibitor or an NS3/4A PI. The current HCV treatment guidelines, a partnership between the American Association for the Study of Liver Diseases (AALSD) and the Infectious Diseases Society of America (IDSA), recommend GLE/PIB for TN HCV GT 1-6-infected patients without cirrhosis and with compensated cirrhosis, as Class 1 , Level A evidence. ${ }^{59}$ As noted earlier, it may be more prudent to treat GT 3 patients for 12 weeks even in the absence of cirrhosis should difficultto-treat characteristics be present. For TE patients, the strength of recommendation ranges from Class 1, Level A, to Class 2, Level B, for patients with prior ribavirin/peginterferon exposure, or GT 1 and 2 with prior DAA failure. However, it is not recommended for patients with GT 3-6 with prior DAA failure, with the regimen of sofosbuvir, velpatasvir, and voxilaprevir being favored (Table 1). ${ }^{53}$ The European Association for the Study of the Liver (EASL) 2018 Guidelines also endorse that TN and TE patients without or with cirrhosis (Child-Pugh A) can be treated with GLE/PIB for 12 weeks without testing GT. If cirrhosis can be reliably excluded by means of a noninvasive marker in TN patients, GLE/PIB can be administered for 8 weeks only. ${ }^{60}$
Future studies should examine GLE/PIB efficacy in GT 2-6 patients with prior NS 3/4a PI or NS5a failures, in children, in more diverse population, and decompensated cirrhotic patients. Given the effectiveness of 8-week therapy, a shorter duration of therapy may be even possible, which would have benefits in terms of compliance and cost.

\section{Conclusion}

Glecaprevir/pibrentasvir has proven to be a highly effective pan-genotypic treatment for HCV in patients without cirrhosis and with compensated cirrhosis, with a high barrier to resistance, and is safe and effective in patients with advanced renal disease, HIV, and solid organ transplants. Moreover, it has high success rates in PRS-TE patients and in patients who have either received prior NS3/4A PIs or NS5A inhibitor therapies; however, its effectiveness is noticeably lower in patients who have previously failed both NS3/4A PIs and NS5A inhibitor therapies. The WHO has put HCV elimination high on the world health agenda. The use of pan-genotypic DAA regimens, such as GLE/PIB, has the potential to support the worldwide goal of HCV elimination.

\section{Disclosure}

Dr Donald M Jensen reports grants from Abbvie, during the conduct of the study; received travel reimbursements from AASLD for attending annual meetings and visiting industry clients, outside the submitted work. The authors report no other conflicts of interest in this work.

\section{References}

1. Polaris Observatory HCV Collaborators. Global prevalence and genotype distribution of hepatitis $\mathrm{C}$ virus infection in 2015: a modelling study. Lancet Gastroenterol Hepatol. 2017;2:161-176. doi:10.1016/ S2468-1253(16)30181-9

2. AASLD-IDSA Guidance Panel. Hepatitis C guidance: AASLD-IDSA recommendations for testing, managing, and treating adults infected with hepatitis C virus. Hepatology. 2015;62:932-954. doi:10.1002/ hep. 27950

3. Ward JW, Hinman AR. What is needed to eliminate hepatitis B virus and hepatitis $\mathrm{C}$ virus as global health threats. Gastroenterology. 2019;156(2):297-310.

4. World Health Organization (WHO). Global health sector strategy on viral hepatitis, 2016-2021. Available from: http://apps.who.int/iris/bit stream/handle/10665/246177/WHO-HIV-2016.06-eng.pdf?sequence= 1. Accessed January 15, 2016

5. Edlin BR, Eckhardt BJ, Shu MA, et al. Toward a more accurate estimate of the prevalence of hepatitis $\mathrm{C}$ in the United States. Hepatology. 2015;62:1353-1363. doi:10.1002/hep.27978

6. Kim WR, Lake JR, Smith JM, et al. OPTN/SRTR 2016 annual data report: liver. Am J Transplant. 2018;18 Suppl 1:172-253. doi:10.1111/ ajt. 14559

7. Cotter TG, Paul S, Sandıkçı B, et al. Improved graft survival after liver transplantation for recipients with hepatitis $\mathrm{C}$ in the direct-acting antiviral era. Liver Transpl. 2019. doi:10.1002/1t.25424 
8. Cotter TG, Paul S, Sandıkçı B, et al. Increasing utilization and excellent initial outcomes following liver transplant of HCV-viremic donors into HCV-negative recipients. Hepatology. 2019. doi:10.1002/hep.30540

9. Belli LS, Perricone G, Adam R, et al. Impact of DAAs on liver transplantation: major effects on the evolution of indications and results. An ELITA study based on the ELTR registry. J Hepatol. 2018;69:810-817. doi:10.1016/j.jhep.2018.06.010

10. Smith DB, Bukh J, Kuiken C, et al. Expanded classification of hepatitis $\mathrm{C}$ virus into 7 genotypes and 67 subtypes: updated criteria and genotype assignment web resource. Hepatology. 2014;59:318327. doi:10.1002/hep. 26744

11. Bukh J. The history of hepatitis $\mathrm{C}$ virus (HCV): basic research reveals unique features in phylogeny, evolution and the viral life cycle with new perspectives for epidemic control. J Hepatol. 2016;65:S2-S21. doi:10.1016/j.jhep.2016.07.035

12. Jopling CL, Yi M, Lancaster AM, Lemon SM, Sarnow P. Modulation of hepatitis $\mathrm{C}$ virus RNA abundance by a liver-specific MicroRNA. Science. 2005;309:1577-1581. doi:10.1126/science.1113329

13. Radkowski M, Gallegos-Orozco JF, Jablonska J, et al. Persistence of hepatitis $\mathrm{C}$ virus in patients successfully treated for chronic hepatitis C. Hepatology. 2005;41:106-114. doi:10.1002/hep.20518

14. Messina JP, Humphreys I, Flaxman A, et al. Global distribution and prevalence of hepatitis C virus genotypes. Hepatology. 2015;61:7787. doi:10.1002/hep. 27259

15. Gower E, Estes C, Blach S, Razavi-Shearer K, Razavi H. Global epidemiology and genotype distribution of the hepatitis $\mathrm{C}$ virus infection. J Hepatol. 2014;61:S45-S57. doi:10.1016/j.jhep.2014.07.027

16. Sievert W, Altraif I, Razavi HA, et al. A systematic review of hepatitis $\mathrm{C}$ virus epidemiology in Asia, Australia and Egypt. Liver Int. 2011;31(Suppl 2):61-80. doi:10.1111/j.1478-3231.2011.02540.x

17. Simmonds P. The origin of hepatitis C virus. Curr Top Microbiol Immunol. 2013;369:1-15. doi:10.1007/978-3-642-27340-7_1

18. Honda M, Ping LH, Rijnbrand RC, et al. Structural requirements for initiation of translation by internal ribosome entry within genomelength hepatitis C virus RNA. Virology. 1996;222:31-42. doi:10.1006/viro.1996.0395

19. von Hahn T, Yoon JC, Alter H, et al. Hepatitis C virus continuously escapes from neutralizing antibody and T-cell responses during chronic infection in vivo. Gastroenterology. 2007;132:667-678. doi:10.1053/j.gastro.2006.12.008

20. Wolk B, Sansonno D, Krausslich HG, et al. Subcellular localization, stability, and trans-cleavage competence of the hepatitis C virus NS3NS4A complex expressed in tetracycline-regulated cell lines. $J$ Virol. 2000;74:2293-2304. doi:10.1128/jvi.74.5.2293-2304.2000

21. Masaki T, Suzuki R, Murakami K, et al. Interaction of hepatitis C virus nonstructural protein $5 \mathrm{~A}$ with core protein is critical for the production of infectious virus particles. J Virol. 2008;82:7964-7976. doi:10.1128/JVI.00826-08

22. Jacobson IM, McHutchison JG, Dusheiko G, et al. Telaprevir for previously untreated chronic hepatitis $\mathrm{C}$ virus infection. $N$ Engl J Med. 2011;364:2405-2416. doi:10.1056/NEJMoa1012912

23. Poordad F, McCone J Jr, Bacon BR, et al. Boceprevir for untreated chronic HCV genotype 1 infection. N Engl J Med. 2011;364:11951206. doi:10.1056/NEJMoa1010494

24. Gordon SC, Muir AJ, Lim JK, et al. Safety profile of boceprevir and telaprevir in chronic hepatitis $\mathrm{C}$ : real world experience from $\mathrm{HCV}$ TARGET. J Hepatol. 2015;62:286-293. doi:10.1016/j.jhep.2014.08.052

25. Jacobson IM, Dore GJ, Foster GR, et al. Simeprevir with pegylated interferon alfa $2 \mathrm{a}$ plus ribavirin in treatment-naive patients with chronic hepatitis $\mathrm{C}$ virus genotype 1 infection (QUEST-1): a phase 3, randomised, double-blind, placebo-controlled trial. Lancet. 2014;384:403-413. doi:10.1016/S0140-6736(14)60494-3

26. Lawitz E, Mangia A, Wyles D, et al. Sofosbuvir for previously untreated chronic hepatitis $\mathrm{C}$ infection. $N$ Engl $J$ Med. 2013;368:1878-1887. doi:10.1056/NEJMoa1214853
27. Kwo P, Gitlin N, Nahass R, et al. Simeprevir plus sofosbuvir (12 and 8 weeks) in hepatitis $\mathrm{C}$ virus genotype 1 -infected patients without cirrhosis: OPTIMIST-1, a phase 3, randomized study. Hepatology. 2016;64:370-380. doi:10.1002/hep.28467

28. Sulkowski MS, Vargas HE, Di Bisceglie AM, et al. Effectiveness of simeprevir plus sofosbuvir, with or without ribavirin, in real-world patients with HCV genotype 1 infection. Gastroenterology. 2016;150:419-429. doi:10.1053/j.gastro.2015.10.013

29. Saxena V, Nyberg L, Pauly M, et al. Safety and efficacy of simeprevir/ sofosbuvir in hepatitis $\mathrm{C}$-infected patients with compensated and decompensated cirrhosis. Hepatology. 2015;62:715-725. doi:10.1002/ hep. 27922

30. Afdhal N, Zeuzem S, Kwo P, et al. Ledipasvir and sofosbuvir for untreated HCV genotype 1 infection. N Engl J Med. 2014;370:18891898. doi:10.1056/NEJMoa1402454

31. Afdhal N, Reddy KR, Nelson DR, et al. Ledipasvir and sofosbuvir for previously treated HCV genotype 1 infection. $N$ Engl J Med. 2014;370:1483-1493. doi:10.1056/NEJMoa1316366

32. Ahmed A, Felmlee DJ. Mechanisms of hepatitis $\mathrm{C}$ viral resistance to direct acting antivirals. Viruses. 2015;7:6716-6729. doi:10.3390/v7122968

33. Ogawa E, Furusyo N, Nomura H, et al. NS5A resistance-associated variants undermine the effectiveness of ledipasvir and sofosbuvir for cirrhotic patients infected with HCV genotype 1b. $J$ Gastroenterol. 2017;52:845-854. doi:10.1007/s00535-016-1290-1

34. Akuta N, Sezaki H, Suzuki F, et al. Ledipasvir plus sofosbuvir as salvage therapy for $\mathrm{HCV}$ genotype 1 failures to prior NS5A inhibitors regimens. $J$ Med Virol. 2017;89:1248-1254. doi: 10.1002/jmv. 24767

35. Kawakami Y, Ochi H, Hayes $\mathrm{CN}$, et al. Efficacy and safety of ledipasvir/sofosbuvir with ribavirin in chronic hepatitis $\mathrm{C}$ patients who failed daclatasvir/asunaprevir therapy: pilot study. $J$ Gastroenterol. 2018;53:548-556. doi:10.1007/s00535-017-1380-8

36. Lafferty L, Treloar C, Guthrie J, Chambers GM, Butler T. Social capital strategies to enhance hepatitis $\mathrm{C}$ treatment awareness and uptake among men in prison. J Viral Hepat. 2017;24:111-116. doi:10.1111/jvh.12627

37. Abbvie Inc. MAVYRET - full prescribing information. 2018. Available from: https://www.rxabbvie.com/pdf/mavyret_pi.pdf. Accessed October 20, 2018

38. Ng TI, Krishnan P, Pilot-Matias T, et al. In vitro antiviral activity and resistance profile of the next-generation hepatitis C virus NS5A inhibitor pibrentasvir. Antimicrob Agents Chemother. 2017;61. doi:10.1128/AAC.02558-16

39. Gao M. Antiviral activity and resistance of HCV NS5A replication complex inhibitors. Curr Opin Virol. 2013;3:514-520. doi:10.1016/j. coviro.2013.06.014

40. Zeuzem S, Foster GR, Wang S, et al. Glecaprevir-pibrentasvir for 8 or 12 weeks in HCV genotype 1 or 3 infection. $N$ Engl J Med. 2018;378:354-369. doi:10.1056/NEJMoa1702417

41. Asselah T, Kowdley KV, Zadeikis N, et al. Efficacy of glecaprevir/pibrentasvir for 8 or 12 weeks in patients with hepatitis $\mathrm{C}$ virus genotype $2,4,5$, or 6 infection without cirrhosis. Clin Gastroenterol Hepatol. 2018;16:417-426. doi:10.1016/j. cgh.2017.09.027

42. Kwo PY, Poordad F, Asatryan A, et al. Glecaprevir and pibrentasvir yield high response rates in patients with HCV genotype 1-6 without cirrhosis. J Hepatol. 2017;67:263-271. doi:10.1016/j.jhep.2017.03.039

43. Forns X, Lee SS, Valdes J, et al. Glecaprevir plus pibrentasvir for chronic hepatitis $\mathrm{C}$ virus genotype $1,2,4,5$, or 6 infection in adults with compensated cirrhosis (EXPEDITION-1): a single-arm, openlabel, multicentre phase 3 trial. Lancet Infect Dis. 2017;17:10621068. doi:10.1016/S1473-3099(17)30496-6

44. Poordad F, Felizarta F, Asatryan A, et al. Glecaprevir and pibrentasvir for 12 weeks for hepatitis $\mathrm{C}$ virus genotype 1 infection and prior direct-acting antiviral treatment. Hepatology. 2017;66:389-397. doi:10.1002/hep. 29081 
45. Poordad F, Pol S, Asatryan A, et al. Glecaprevir/pibrentasvir in patients with hepatitis $\mathrm{C}$ virus genotype 1 or 4 and past direct-acting antiviral treatment failure. Hepatology. 2018;67:1253-1260. doi:10.1002/hep.29671

46. Gane E, Lawitz E, Pugatch D, et al. Glecaprevir and pibrentasvir in patients with $\mathrm{HCV}$ and severe renal impairment. $N$ Engl J Med. 2017;377:1448-1455. doi:10.1056/NEJMoa1704053

47. Rockstroh JK, Lacombe K, Viani RM, et al. Efficacy and safety of glecaprevir/pibrentasvir in patients coinfected with hepatitis $\mathrm{C}$ virus and human immunodeficiency virus type 1: the EXPEDITION-2 study. Clin Infect Dis. 2018;67:1010-1017. doi:10.1093/cid/ciy220

48. Reau N, Kwo PY, Rhee S, et al. Glecaprevir/pibrentasvir treatment in liver or kidney transplant patients with hepatitis $\mathrm{C}$ virus infection. Hepatology. 2018. doi:10.1002/hep.30046

49. Asselah T, Lee SS, Yao BB, et al. Efficacy and safety of glecaprevir/ pibrentasvir in patients with chronic hepatitis $\mathrm{C}$ virus genotype 5 or 6 infection (ENDURANCE-5,6): an open-label, multicentre, phase $3 \mathrm{~b}$ trial. Lancet Gastroenterol Hepatol. 2019;4:45-51. doi:10.1016/ S2468-1253(18)30341-8

50. Foster GR, Asselah T, Kopecky-Bromberg S, et al. Safety and efficacy of glecaprevir/pibrentasvir for the treatment of chronic hepatitis C in patients aged 65 years or older. PLoS One. 2019;14:e0208506. doi:10.1371/journal.pone.0208506

51. D'Ambrosio R, Pasulo L, Puoti M, et al. Real-world effectiveness and safety of glecaprevir/pibrentasvir in 723 patients with chronic hepatitis C. J Hepatol. 2018. doi:10.1016/S0168-8278(18)30351-9

52. Flamm S, Mutimer D, Asatryan A, et al. Glecaprevir/pibrentasvir in patients with chronic HCV genotype 3 infection: an integrated phase 2/3 analysis. J Viral Hepat. 2018. doi:10.1111/jvh.13038

53. Bourliere M, Gordon SC, Flamm SL, et al. Sofosbuvir, velpatasvir, and voxilaprevir for previously treated HCV infection. $N$ Engl J Med. 2017;376:2134-2146. doi:10.1056/NEJMoa1613512
54. Osawa M, Imamura M, Teraoka Y, et al. Real-world efficacy of glecaprevir plus pibrentasvir for chronic hepatitis $\mathrm{C}$ patient with previous direct-acting antiviral therapy failures. $J$ Gastroenterol. 2019;54 (3):291-296.

55. Uemura H, Uchida Y, Kouyama JI, et al. NS5A-P32 deletion as a factor involved in virologic failure in patients receiving glecaprevir and pibrentasvir. J Gastroenterol. 2019;54:459-470. doi:10.1007/ s00535-018-01543-9

56. Krishnan P, Pilot-Matias T, Schnell G, et al. Pooled resistance analysis in patients with hepatitis $\mathrm{C}$ virus genotype 1 to 6 infection treated with glecaprevir-pibrentasvir in phase 2 and 3 clinical trials. Antimicrob Agents Chemother. 2018;62. doi:10.1128/ AAC.01249-18

57. Wyles DWO, Yao B, Reindollar RW, Weilert F, Dufour J-F. Retreatment of hepatitis $\mathrm{C}$ virus infection in patients who failed glecaprevir/pibrentasvir. $J \quad$ Hepatol. 2018;68:S623-S624. doi:10.1016/S0168-8278(18)30265-4

58. Dhiman RK, Grover GS, Premkumar M, et al. Direct-acting antiviral therapy is safe and effective in pediatric chronic hepatitis $\mathrm{C}$ : the public health perspective. $J$ Pediatr Gastroenterol Nutr. 2019;68:74-80. doi:10.1097/MPG.0000000000002139

59. AASLD-IDSA. HCV Guidance: Recommendations for Testing, Managing, and Treating Hepatitis C; Clin Infect Dis. 2018;30;67 (10):1477-1492.

60. European Association for the Study of the Liver. Electronic address eee, European Association for the Study of the L. EASL recommendations on treatment of hepatitis C 2018. J Hepatol. 2018;69:461511. doi:10.1016/j.jhep.2018.03.026
Drug Design, Development and Therapy

\section{Publish your work in this journal}

Drug Design, Development and Therapy is an international, peerreviewed open-access journal that spans the spectrum of drug design and development through to clinical applications. Clinical outcomes, patient safety, and programs for the development and effective, safe, and sustained use of medicines are a feature of the journal, which has also

\section{Dovepress}

been accepted for indexing on PubMed Central. The manuscript management system is completely online and includes a very quick and fair peer-review system, which is all easy to use. Visit http://www. dovepress.com/testimonials.php to read real quotes from published authors. 